\title{
Enthesopathy of the pectoralis major tendon mimicking osteoid osteoma. A case report with an unfortunate series of events
}

\author{
Daniel Baumhoer ${ }^{1}$ \\ Gernot Jundt ${ }^{1}$ \\ Ulrich Lenze ${ }^{2}$ \\ Clemens Reisinger ${ }^{3}$ \\ Anna Hirschmann ${ }^{3}$
}

1 Bone Tumor Reference Center at the Institute of Pathology University Hospital Basel, Switzerland

2 Orthopaedic Department Basel University Childrens Hospital (UKBB), Switzerland

${ }^{3}$ Department of Radiology and Nuclear Medicine, University of Basel Hospital, Switzerland

Corresponding author:

Anna Hirschmann

Department of Radiology and Nuclear Medicine, University of Basel Hospital

Petersgraben 4

4031 Basel, Switzerland

E-mail: anna.hirschmann@usb.ch

\section{Summary}

Background: we present the case of an enthesopathy at the proximal humerus which was initially - due to the clinical history and a positive bone scintigraphy - regarded suspicious for metastatic breast cancer in a 50-year-old woman.

Case report: after complementing radiographs and a magnetic resonance (MR) examination exhibiting a focally contrast enhancing juxtacortical osteolysis of the humerus, a metastasis seemed radiologically unlikely, but besides a traction-related periosteal reaction of the pectoralis major tendon an unusual osteoid osteoma could not unequivocally be ruled out. Although radiological follow-up was recommended the patient insisted on a surgical resection that was performed subsequently and confirmed an enthesopathy. Shortly after, she fractured her upper arm following minor trauma but is doing well after conservative treatment since then. Conclusion: enthesopathies presenting as unusual periosteal reactions can mimic primary and secondary bone tumors and should always be included in the differential diagnosis.

KEY WORDS: bone scan, enthesopathy, metastases, osteoid osteoma, pectoralis major tendon, secondary fracture.

\begin{abstract}
Introduction
Tendons, aponeuroses and fasciae transfer the forces developed by muscle activity to bone and are firmly attached at both the periosteum and the cortical bone by extrinsic collagen fibers ${ }^{1}$. Summarized under the term entheses, these bone-soft tissue interfaces are especially prone to mechanical injuries that are well documented in a number of sports. As a consequence of repetitive biomechanical stress affecting the periosteal anchorage, extensive reactive new bone formation can occur and clinically as well as radiologically mimic neoplastic disease. We present a case of an enthesopathy of the pectoralis major tendon that was initially regarded suspicious for a metastasis, then, during radiological workup, proved difficult to distinguish from an osteoid osteoma and eventually was resected. The report illustrates the difficulties in assessing enthesopathies with unusual presentations and the potential complications of avoidable partial resections of cortical bone.
\end{abstract}

\section{Case report}

A 50-year-old woman with a history of localized breast cancer (TNM classification: pT1c, pNo, cM0, G2) that was resected and treated with additional adjuvant radiotherapy three years ago, underwent a $99 \mathrm{~m}$ Technetium DPD bone scintigraphy due to chronic back pain to assess bone metastasis. While the spine was unremarkable, the left proximal humerus showed a focal uptake of the radioisotope on the whole body scan and the spot view of the left upper arm (Fig. 1). Specifically asked for symptoms she complained about minor pain in this region. Due to clinically suspected metastatic breast cancer, radiographs and a magnetic resonance (MR) imaging were acquired. The externally rotated anteroposterior radiograph of the left proximal humerus showed a focal juxtacortical osteolysis of $8 \times 5 \mathrm{~mm}$ with a central calcification of the lateral cortex and a concomitant uniform smooth, non-aggressive periosteal reaction (Fig. 2). Corresponding to the radiographs, the MR images revealed a $5 \times 4 \mathrm{~mm}$ lesion adjacent to the anterolateral humeral cortex with increased signal intensity on the T2-weighted fat saturated images, intermediate signal intensity on the T1-weighted images and enhancement after contrast administration (Fig. 3 A-D). The cortex underneath the lesion was slightly thinned, neither bone nor soft tis- 


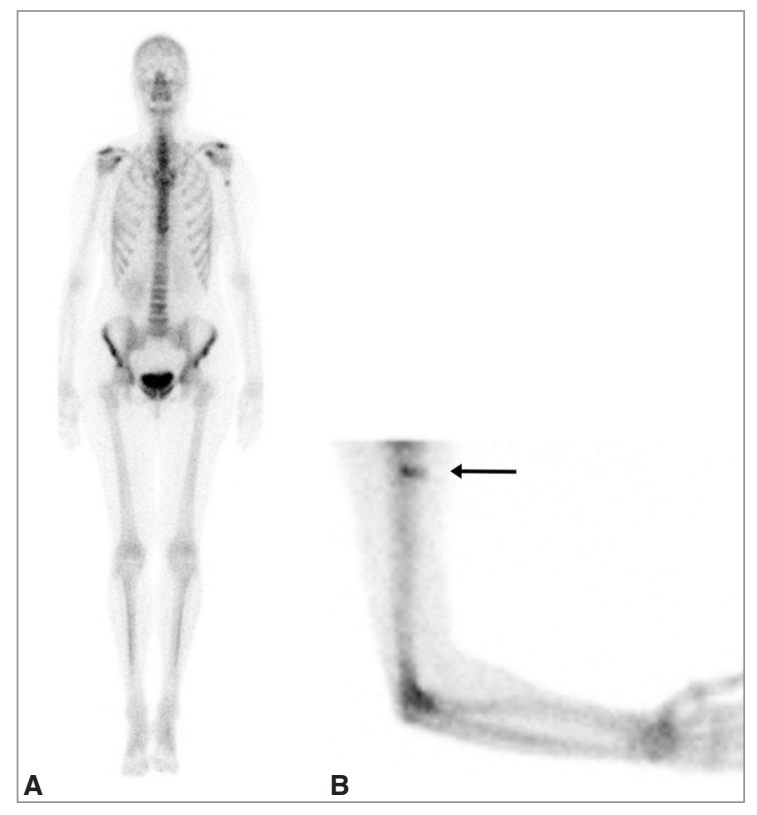

Figure 1. A 50-year-old woman with chronic back pain and clinical history of breast cancer three years ago underwent a 99m Technetium DPD bone scintigraphy with a focal bone tracer uptake (arrow) of the left proximal lateral humerus shown on the whole body scan $(A)$ as well as on the spot view $(\mathrm{B})$.

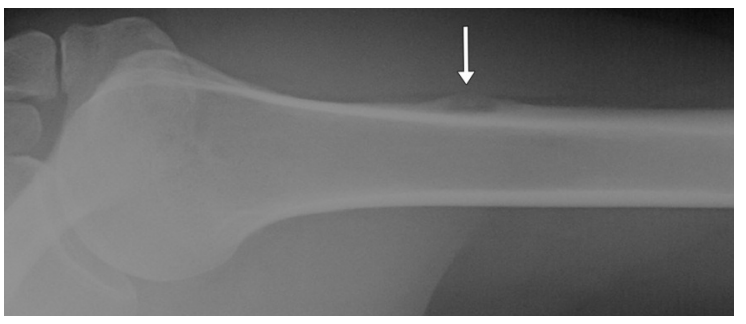

Figure 2. External rotation anteroposterior radiograph of the left humerus shows a focal juxtacortical lucency (arrow) surrounded by a smooth, uniform non-aggressive periosteal reaction.

sue edema were evident perifocally. The insertion of the pectoralis major tendon was immediately adjacent to the lesion and the tendon itself seemed unremarkable (Fig. $3 \mathrm{C}$ ). Subsequently, the case was presented and discussed at an international and interdisciplinary meeting of radiologists, pathologists and orthopaedic surgeons on bone tumors and the majority of participating radiologists agreed that the lesion most likely represented an enthesopathy of the pectoralis major tendon at its attachment to the humerus. However, an osteoid osteoma was still regarded as a rather unlikely but possible differential diagnosis. Although the committee of the conference recommended a clinical and radiological follow-up in six months, the patient nevertheless insisted on a resection of the lesion fearing metastastic breast cancer. A partial cortical resection with a safety margin of $1.5 \mathrm{~cm}$ around the palpable mass was performed
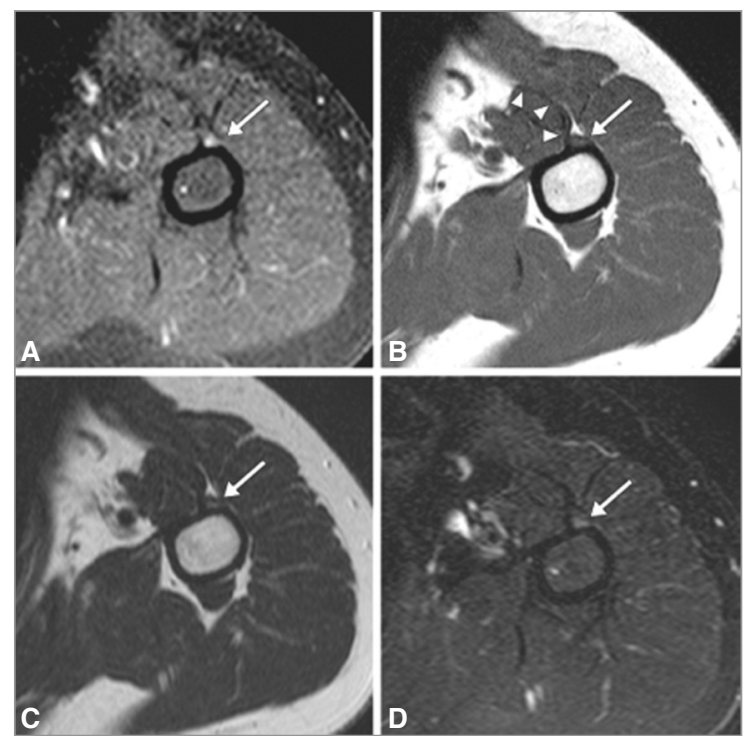

Figure 3. MR images show a small, $5 \times 4 \mathrm{~mm}$ juxtacortical lesion (arrows) of the anterolateral humerus at the insertion of the pectoralis major tendon (arrowheads in B) with increased signal intensity on the T2 fat suppressed image (A), slight increased signal intensity on the T2 image (B), intermediate signal intensity on the T1 image (C) and enhancement after contrast administration (D). The underyling cortex is slightly thinned.

subsequently. Macroscopically, the specimen measured $40 \times 14 \times 7 \mathrm{~mm}$ and consisted mainly of cortical bone with only minimal soft tissue attached. On cut section, a subperiosteal bony outgrowth $(12 \times 6 \times$ $5 \mathrm{~mm}$ ) with broad connection to the underlying cortex was revealed showing insertion of tendon fibres at its proximal end. Histologically, the lesion consisted of fibroblastic tissue rich in capillary vessels and activated osteoblasts synthesizing an immature osteoid matrix (Fig. 4). There was therefore indeed considerable morphologic overlap to the characteristic findings of an osteoid osteoma although the zonal pattern and architecture consisting of a central nidus and a peripheral rim of sclerotic bone were missing. Instead, the lesion encased fragments of lamellar bone with empty osteocyte lacunae indicating prior and potentially causative traumatization (Fig. 4). Active remodelling including deposition of newly formed bone onto devitalized fragments but also osteoclastic resorption was observed throughout the lesion. No cellular atypia or metastatic infiltrates were identified, ruling out metastatic breast cancer or a primary bone tumor. The tendon fibres showing chondroid metaplasia inserted directly at the proximal end of the lesion and the diagnosis of an enthesopathy was made.

Four weeks after the resection, the patient stumbled, fall on her left arm and fractured her humerus at the site of resection due to an abrupt external rotation movement. The fracture was treated conservatively and healed without further complications. The patient is doing well since 24 months. 


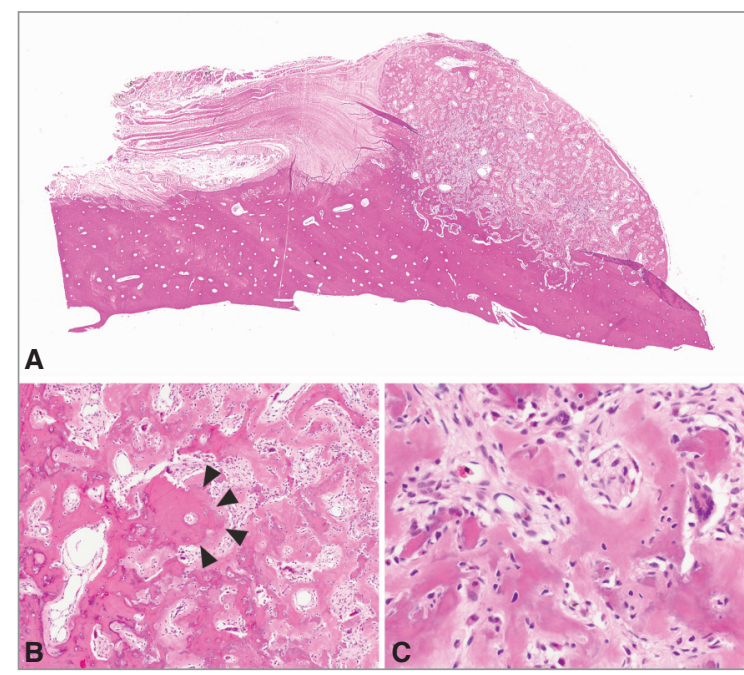

Figure 4. Histology of the resection specimen revealed an osteoid osteoma-like reactive new bone formation directly at the insertion of the pectoralis major tendon $(A, H \& E$ stain, $x 1$ magnification). Higher magnification shows residual lamellar bone (arrowheads) surrounded by irregular woven bone (B, H\&E stain, x50 magnification) and a well vascularized fibrous stroma without atypical cells (C, H\&E stain, x200 magnification).

\section{Discussion}

Due to their site-specific and functional properties, entheses are especially vulnerable to increased mechanical stress which is well known in acute or overuse injuries in sports ${ }^{2}, 3$. Since traumatic enthesopathies are usually diagnosed clinically and radiologically without major difficulties, surgical resection is exceptional and, thus, not much is known about the histopathology of those lesions.

In the case presented here, a positive bone scan in the context of a history of breast cancer initially suggested a metastasis at the proximal humerus but subsequent radiographs and MR imaging strongly argued against this differential diagnosis. Instead, an osteoid osteoma was discussed due to the juxtacortical localisation and the positive bone scan. Although only minor pain was reported by the patient and the characteristic perifocal edema was not evident, the absence of these findings does not exclude the diagnosis, especially with increasing age in which these reactive changes have been reported to sometimes occur to a lesser extent ${ }^{4-6}$. An enthesopathy, however, was considered the most likely differential diagnosis. The pectoralis major tendon inserts at the lateral lip of the bicipital groove of the humerus, directly adjacent to the location of the small focus of signal abnormality presented here ${ }^{7}$. Injuries to the pectoralis major tendon are rare and occur most often at the site of insertion ${ }^{7,8}$. Tears as well as calcific tendinitis are the most common pathological findings of the pectoralis tendon ${ }^{9-11}$ whereas chronic avulsion injuries more frequently involve the deltoid tendon in- serting at the humerus or the gluteus maximus tendon at the femur ${ }^{12,13}$

Histologically, the reactive nature of the lesion was confirmed and a primary or secondary bone tumor could be ruled out with certainty. The fragments of necrotic lamellar bone and the tendon fibres directly inserting at and merging with the lesion indicated a causative traction injury. The patient played golf casually and since she could not recall a singular trauma the lesion might have developed due to iterative minor stress. Absence of abnormal periosteal signal intensity on MR images also encouraged causative chronic micro-injuries over an acute tendon pathology. The lesion could therefore be embraced as a pseudotumor pectoralis ${ }^{7,13}$.

Taken together, enthesopathy should always be included in the differential diagnosis of unusual cortical reactions although prior traumatization is not necessarily evident. Not surprisingly, furthermore, cortical resections affect the biomechanical stability of bones and can result in avoidable pathological fractures.

\section{Ethical standards}

The Authors state that the case reported here meets the ethical standards of the Journal ${ }^{14}$.

\section{References}

1. Standring S. Functional anatomy of the muskuloskeletal system. In: Standring S, ed. Gray's Anatomy - The Anatomical Basis of Clinical Practice. 39th ed. London, UK: Elsevier Churchill Livingstone. 2005:83-136.

2. Benjamin M, Toumi H, Ralphs JR, Bydder G, Best TM, Milz S. Where tendons and ligaments meet bone: attachment sites ('entheses') in relation to exercise and/or mechanical load. J Anat. 2006;208:471-490.

3. Arend CF. Role of sonography and magnetic resonance imaging in detecting deltoideal acromial enthesopathy: an early finding in the diagnosis of spondyloarthritis and an under-recognized cause of posterior shoulder pain. $\mathrm{J}$ Ultrasound Med. 2014;33:557-561.

4. Assoun J, Richardi G, Railhac JJ, et al. Osteoid osteoma: MR imaging versus CT. Radiology. 1994;191:217-223.

5. Davies M, Cassar-Pullicino VN, Davies AM, McCall IW, Tyrrell $\mathrm{PN}$. The diagnostic accuracy of MR imaging in osteoid osteoma. Skeletal Radiol. 2002;31:559-569.

6. Ehara S, Rosenthal DI, Aoki J, et al. Peritumoral edema in osteoid osteoma on magnetic resonance imaging. Skeletal Radiol. 1999;28:265-270.

7. Connell DA, Potter HG, Sherman MF, Wickiewicz TL. Injuries of the pectoralis major muscle: evaluation with MR imaging. Radiology. 1999;210:785-791.

8. Merolla G, Paladini P, Campi F, Porcellini G. Pectoralis major tendon rupture. Surgical procedures review. Muscles Ligaments Tendons J. 2012;2:96-103.

9. Durr HR, Lienemann A, Silbernagl H, Nerlich A, Refior HJ. Acute calcific tendinitis of the pectoralis major insertion associated with cortical bone erosion. Eur Radiol. 1997;7:1215-1217.

10. Chadwick CJ. Tendinitis of the pectoralis major insertion with humeral lesions. A report of two cases. J Bone Joint Surg Br. 1989;71:816-818.

11. Flemming DJ, Murphey MD, Shekitka KM, Temple HT, Jelinek 
JJ, Kransdorf MJ. Osseous involvement in calcific tendinitis: a retrospective review of 50 cases. AJR Am J Roentgenol. 2003;181:965-972.

12. Moser T, Lecours J, Michaud J, Bureau NJ, Guillin R, Cardinal E. The deltoid, a forgotten muscle of the shoulder. Skeletal Radiol. 2013;42:1361-1375.
13. Morgan H, Damron T, Cohen H, Allen M. Pseudotumor deltoideus: a previously undescribed anatomic variant at the deltoid insertion site. Skeletal Radiol. 2001;30:512-518.

14. Padulo J, Oliva F, Frizziero A, Maffulli N. Muscles, Ligaments and Tendons Journal. Basic principles and recommendations in clinical and field science research. MLTJ. 2013;4:250-252. 